OPEN ACCESS

Edited by:

Andreas Agathangelidis, Institute of Applied Biosciences (INAB), Greece

Reviewed by:

Riccardo Bomben,

Aviano Oncology Reference Center (IRCCS), Italy

Giovanna Cutrona San Martino Hospital (IRCSS), Italy

*Correspondence:

Domenico Maisano

maisano@unicz.it Vincenzo Dattilo

vdattilo@fatebenefratelli.eu Selena Mimm mimmi@unicz.it

${ }^{\dagger}$ These authors share first authorship

FThese authors share senior authorship

Specialty section

This article was submitted to Hematologic Malignancies, a section of the journal

Frontiers in Oncology

Received: 30 April 2021 Accepted: 31 May 2021

Published: 18 June 2021

Citation:

Maisano $D$, laccino $E$ D'Ambrosio A, Chiurazzi $F$, Dattilo V, Scalise M, Gentile M, Vecchio E, Nisticò N, Aloisio A

De Sensi E, Fiume G.

Quinto I and Mimmi S (2021)

Predominant VH1-69 IgBCR

Clones Show Higher Expression of

CD5 in Heterogeneous Chronic Lymphocytic Leukemia Populations.

Front. Oncol. 11:703254.

doi: 10.3389/fonc.2021.703254

\section{Predominant VH1-69 IgBCR Clones Show Higher Expression of CD5 in Heterogeneous Chronic Lymphocytic Leukemia Populations}

\author{
Domenico Maisano ${ }^{1 * t}$, Enrico laccino ${ }^{1 \dagger}$, Alessandro D'Ambrosio $^{2}$, Federico Chiurazzi ${ }^{2}$, \\ Vincenzo Dattilo ${ }^{3 *}$, Mariangela Scalise ${ }^{4}$, Massimo Gentile ${ }^{5}$, Eleonora Vecchio ${ }^{1}$, \\ Nancy Nistico ${ }^{1}$, Annamaria Aloisio ${ }^{1}$, Erika De Sensi ${ }^{1}$, Giuseppe Fiume ${ }^{1}$, Ileana Quinto ${ }^{1 \neq}$ \\ and Selena Mimmi ${ }^{1 \text { 和 }}$

\footnotetext{
${ }^{1}$ Laboratory of Immunology, Department of Experimental and Clinical Medicine, University Magna Graecia of Catanzaro, Catanzaro, Italy, ${ }^{2}$ Hematological Clinic, Department of Clinical Medicine, University "Federico II" of Naples, Naples, Italy, Cardiology, Department of Experimental and Clinical Medicine, University Magna Graecia of Catanzaro, Catanzaro, Italy, ${ }^{5}$ Hematology Unit, AO, Cosenza, Italy
} \\ ${ }^{3}$ Genetics Unit, IRCCS Centro San Giovanni di Dio Fatebenefratelli, Brescia, Italy, ${ }^{4}$ Laboratory of Molecular and Cellular
}

The immunoglobulin B cell receptor (IgBCR) expressed by chronic lymphocytic leukemia (CLL) B cells plays a pivotal role in tumorigenesis, supporting neoplastic transformation, survival, and expansion of tumor clones. We demonstrated that in the same patient, two or more CLL clones could coexist, recognized by the expression of different variable regions of the heavy chain of IgBCR, composing the antigen-binding site. In this regard, phage display screening could be considered the easier and most advantageous methodology for the identification of small peptide molecules able to mimic the natural antigen of the tumor lgBCRs. These molecules, properly functionalized, could be used as a probe to specifically identify and isolate single CLL subpopulations, for a deeper analysis in terms of drug resistance, phenotype, and gene expression. Furthermore, CLL cells express another surface membrane receptor, the CD5, which is commonly expressed by normal T cells. Piece of evidence supports a possible contribution of CD5 to the selection and maintenance of autoreactivity in B cells and the constitutive expression of CD5 on CLL cells could induce pro-survival stimuli. In this brief research report, we describe a peptidebased single-cell sorting using as bait the IgBCR of tumor cells; in the next step, we performed a quantitative analysis of CD5 expression by qRT-PCR related to the expressed IgBCR. Our approach could open a new perspective for the identification, isolation, and investigation of all subsets of IgBCR-related CLL clones, with particular attention to the more aggressive clones.

Keywords: chronic lymphocytic leukemia, phage display, immunoglobulin B cell receptor, peptide-based sorting, gene expression 


\section{INTRODUCTION}

CD5 is a membrane surface receptor expressed by thymocytes, mature T cells, B1a subset of B cells, and leukemic B cells of chronic lymphocytic leukemia (B-CLL) disease $(1,2)$. Called also Leu-1, it is a $67-\mathrm{kDa}$ type I transmembrane glycoprotein monomer and a member of the scavenger receptor cysteinerich (SRCR) family (3). The extracellular region is composed of three different domains (D1, D2, and D3) and represents the putative binding region, while the intracellular domain contains the Immunoreceptor Tyrosine-based Activation Motif (ITAM) sequence as the docking site for phosphorylated Src homology 2 (SH2) domain-containing proteins (4).

CD5 is not expressed in normal B cells, except the B1 subgroup, while it is mostly expressed in B-CLL cells (2); this suggests a possible critical role of CD5 in self-maintenance and progression of neoplastic B cells $(5,6)$. This hypothesis is supported by the evidence that CD5 activates multiple signaling pathways, including mitogen-activated protein kinase (MAPK) (Ras/Erk) pathway, the $\mathrm{Ca} 2+-$ calmodulin-calcineurin-NFAT pathway, and the PI3-K/Akt/mTOR pathway (7). Further, in transgenic mice, the expression of CD5 correlates with the self-reactivity in B cell populations, supporting a possible contribution to the selection and maintenance of autoreactivity in B cells (8).

CLL is the most frequent adult leukemia in western countries, with a variable clinical course and the occurrence of a heterogeneous tumor population $(9,10)$. Increasing evidence supports the hypothesis that CLL pathogenesis is an antigendriven process by a continuous triggering of the immunoglobulin $B$ cell receptor (IgBCR) (11-15), resulting in the no random choice of heavy chain variable region (VH) family during B cell development and the consequential expression of the stereotyped IgBCRs, frequently found in different patients (16). The expression of peculiar IgBCRs is often related to the aggressiveness of the disease (17). Indeed, the unmutated CLL (U-CLL), with less than $2 \%$ of mutation in comparison with the germline sequence, seems to be more aggressive with respect to the mutated CLL (M-CLL), which shows a higher percentage of mutations in the variable region of the heavy chain (18). Furthermore, in the U-CLL subgroup, the rearrangement VH1-69 is the most representative (about 25\%), and patients showed an aggressive disease with the expansion of CLL clones expressing the unmutated IgBCRs, drug resistance, and often a fatal outcome $(16,19)$.

Since CD5 seems to be located close to the surface IgBCR on the B cell surface (6) and seems to be a potential ligand of peculiar Ig heavy chain framework sequences in malignant B cells (5), these findings suggest that CD5 could be a self-antigen recognized by the CLL-IgBCRs, promoting survival and proliferation signaling.

In our last published work, we analyzed two CLL patients (named CLL1 and CLL5) for 2-years observation, demonstrating the coexisting of several leukemic subpopulations identified by different IgBCRs, but the most representative subpopulation identified by the rearrangement VH1-69 persisted during all the time (20). So, based on the evidence mentioned above, we asked whether the survival and progression of the VH1-69 subpopulation could be related to higher CD5 gene expression levels compared to the other coexisting clones. Taking advantage of the previously selected peptide (named p1), able to specifically target the leukemic cells expressing the rearrangement VH1-69 (20), we performed a peptide-based cell sorting, in order to isolate the VH1-69 clones from peripheral blood of both oligoclonal CLL1 and CLL5 patients. p1 positive sorted clones (corresponding to the VH1-69 clones) were analyzed by qRTPCR for the expression of CD5, compared to the other CLL clones ( $\mathrm{p} 1$ negative clones).

Our results demonstrate that this approach, extended to all IgBCR subsets, could open new strategies for a deeper comprehension of the most aggressive clones, analyzing a wide range of molecular mechanisms and drug resistance - related genes.

\section{METHODS}

\section{Peptide-Based Cell Sorting}

Frozen B-CLL cells previously isolated from CLL patients were gently thawed and $24 \mathrm{~h}$-cultured in RPMI medium supplemented with $10 \%$ fetal bovine serum. Then, cells $\left(1 \times 10^{7}\right.$ cells $)$ were first labelled with anti CD19-APC (Miltenyi Biotec, Germany, cat.n. 170-078-090) and anti-CD5-PE. (Miltenyi Biotec - Germany, cat.n. 130-110-990) antibodies, for setting the gate of B-CLL cells. Bulk CLL cells were incubated with FITC-conjugated peptide $\mathrm{p} 1$ $(1 \mathrm{ng} / \mathrm{ml})$ at $4^{\circ} \mathrm{C}$ for $20 \mathrm{~min}$, analyzed by flow cytometry, and $\mathrm{p} 1-$ positive cells were sorted by BD FACSAria III TM (Becton Dickinson). Gating was done using the BD FACSDiva ${ }^{\mathrm{TM}}$ software (Becton, Dickinson Biosciences). Cell sorting was performed with $70-\mu \mathrm{m}$ nozzle size and sorted directly into 5$\mathrm{ml}$ tubes containing $3 \mathrm{ml}$ of staining media in order to minimize cellular stress. Cells were gated in FSC-A vs SSC-A and single cells gated in FSC-H vs FSC-A. Stringent gating strategies to exclude debris or dead cells that exhibit autofluorescence and CD5 negative cells were applied.

\section{qRT-PCR for CD5 Gene Expression}

Total mRNA was extracted from CLL cells (bulk or sorted cells) by TRIzol RNA Isolation Reagents (Invitrogen) according to the manufacturer's instructions and quantified by spectrophotometer. 500ng of total mRNA was retrotranscribed into cDNA using the iScript ${ }^{\text {TM }}$ cDNA Synthesis Kit (BioRad).

qRT-PCR was performed using the CD5 primers (forward $5^{\prime}$ CAGAAGAAGCAGCGCCAGT 3'; reverse 5' TCCTGGGA GGTTGGCTGTATT $3^{\prime}$ ). The general reaction conditions were as follows: initial denaturation step at $95^{\circ} \mathrm{C}$ for $10 \mathrm{~min} ; 40$ cycles of denaturation at $95^{\circ} \mathrm{C}$ for $10 \mathrm{~s}$; annealing at $57^{\circ} \mathrm{C}$ for $10 \mathrm{~s}$; and elongation at $72^{\circ} \mathrm{C}$. All reactions were performed in triplicate employing the CFX96 Touch Deep Well Real-Time PCR System (BioRad). The results normalized to the GAPDH housekeeping gene and determined by $\Delta \Delta \mathrm{Ct}$ method were represented as log 10 fold expression \pm SD of triplicate assessments. Statistical significance was evaluated using one-way analysis of variance (ANOVA), followed by Bonferroni's test for multiple 
comparisons. Bars show mean values $\pm 95 \%$ confidence intervals based on three biological replications.

\section{RESULTS}

The two CLL patients (named CLL1 and CLL5) were previously analyzed on 2 years observation (20). In particular, the patient CLL1 was analyzed at Binet stage A at months 1 and 5 (CLL1A and CLL1B, respectively) and Binet stage $\mathrm{C}$ at month 8 (CLL1C); the patient CLL5, being Binet stage A all the time, was analyzed at months 1, 12, and 24 (CLL5A, CLL5B, and CLL5C, respectively) (Table 1). As reported by Table 1, the VH1-69 subpopulation persisted all the time, at different percentages of representativeness with respect to the other leukemic clones, related to the aggressiveness of the disease. We firstly analyzed by qRT-PCR the CD5 expression levels in total CD5 positive B-CLL cells of CLL1 and CLL5 patients at different times of disease. As shown in Figure 1A, relative gene expression of CD5 was slightly increased, but not statistically significant comparing CLL1A to CLL1B (both Binet stage A). However, a significant increase in CD5 expression was associated with the expansion of VH1-69 clone in the passage from Binet stage A to C (CLL1A fold 1.00土 0.19 ; CLL1C fold $1.37 \pm 0.06$ ), passing from $50 \%$ to $60 \%$ up to $80 \%$ of representativeness. Differently, in CLL5 patient CD5 expression levels significantly decreased comparing CLL5A and CLL5B, associated to a representativeness decrease of VH1-69 clone, passing from $75 \%$ to $35 \%$; further, relative gene expression of CD5 was slightly decreased, but not statistically significant comparing CLL5B to CLL5C (Figure 1B). These observations suggested that the expression level of CD5 correlated with the percentage of the existing VH1-69 CLL clones.

To deepen our analysis, we took advantage of the previously identified peptide p1 as a specific ligand of the VH1-69 unmutated subpopulation in CLL1 and CLL5 patients (20). Indeed, in this work, we used the peptide $\mathrm{p} 1$ as a probe to sort the VH1-69 subpopulation from the total B-CLL cells of CLL1 and CLL5 patients. Figure 2 shows the coexisting of a p1 positive and a p1 negative population in the total CLL cells of CLL1 (Figure 2A) and CLL5 (Figure 2B) patients. After peptidebased sorting, IgBCRs sequence was analyzed both in p1positive and p1-negative fraction, to validate the sorting procedures. No VH1-69 rearrangement was found in the p1negative fraction.

TABLE 1 | Clinical and molecular data of CLL1 and CLL5 patients.

\begin{tabular}{|c|c|c|c|c|}
\hline Patient & Sample (collection time) & WBC (\% of CD19/CD5 positive) & Binet stage & $\begin{array}{c}\text { VH1-69 subpopulation/tota } \\
\text { CLL cells (\%) }\end{array}$ \\
\hline \multirow[t]{3}{*}{ CLL1 65-years old male } & CLL1A (month 1) & 40,410/mmc (90\%) & A & $60 \%$ \\
\hline & CLL1B (month 5) & 69,070/mmc (92\%) & $A$ & $50 \%$ \\
\hline & CLL1C (month 8) & 92,670/mmc (99\%) & $\mathrm{C}$ & $80 \%$ \\
\hline \multirow[t]{3}{*}{ CLL5 80-years old female } & CLL5A (month 1) & $57,210 / \mathrm{mmc}(95 \%)$ & $A$ & $75 \%$ \\
\hline & CLL5B (month 12) & 119,999/mmc (98\%) & $A$ & $45 \%$ \\
\hline & CLL5C (month 24) & $86,500 / \mathrm{mmc}(96 \%)$ & $A$ & $35 \%$ \\
\hline
\end{tabular}

Peripheral blood samples of CLL1 and CLL5 patients were collected at the indicated time. CLL stage was defined according to Binet classification (Cancer.Net Editorial Board, 10/2017). The percentage of VH1-69 subpopulation cells was determined by IgBCR sequencing as previously reported (20). The nucleotide sequences of CLL IgBCRs were deposited (GenBank accession numbers MT334403 to MT334414).

A

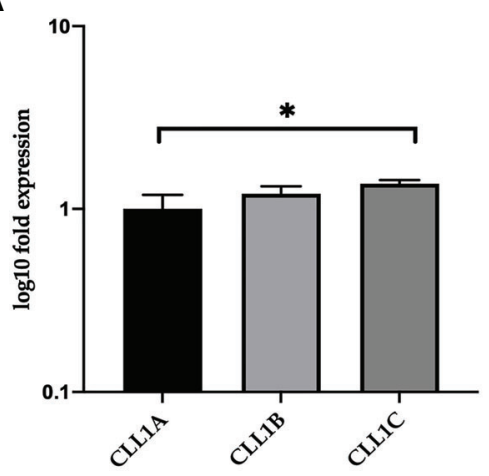

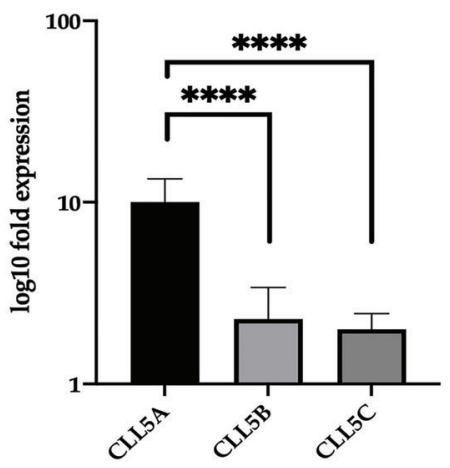

FIGURE 1 | Relative expression of CD5 in CLL clones of CLL1 and CLL5 patients. Relative CD5 gene expression in total B-CLL cells from CLL1 (A) and CLL5 (B) patient. The results were normalized to the GAPDH housekeeping gene, determined by $\Delta \Delta \mathrm{Ct}$ method, and represented as log 10 fold expression $\pm \mathrm{SD}$ of triplicate assessments. Statistical significance was evaluated using one-way analysis of variance (ANOVA), followed by Bonferroni's test for multiple comparisons. Bars show mean values $\pm 95 \%$ confidence intervals based on three biological replications. ${ }^{*} \mathrm{P} \leq 0.01$; ${ }^{\star \star \star \star} P \leq 0.0001$. 
A

CLL1C

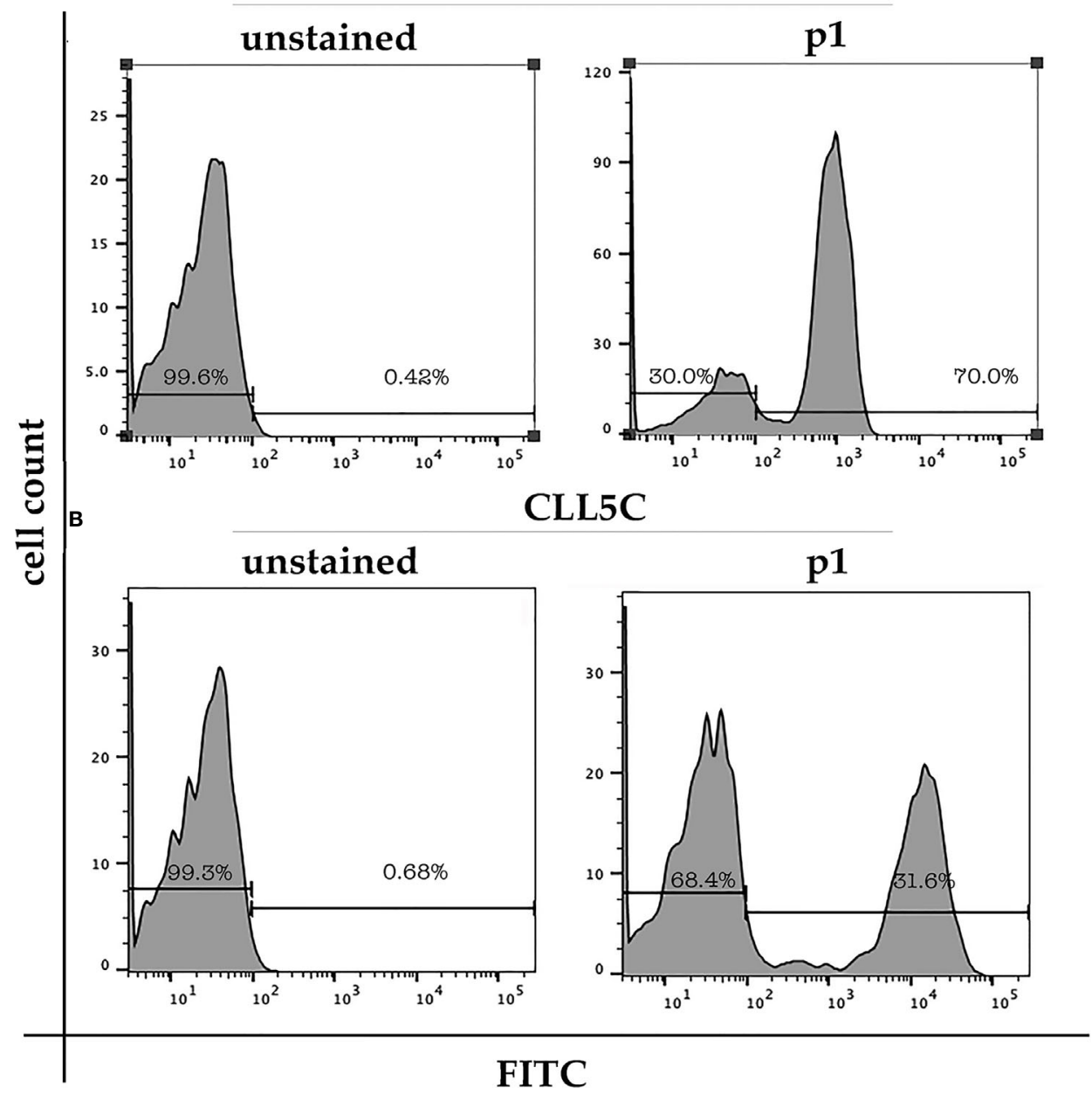

FIGURE 2 | CD19/CD5 positive B-CLL cells isolated from CLL1C (month 8, panel A) and CLL5C (month 24, panel B) patients were stained with FITCconjugated peptide p1 or unstained. p1-positive cells corresponding to the VH 1-69 clones were 70\% of total B-CLL population in CLL1C patient and 32\% in CLL5C patient.

Then, we analyzed by qRT-PCR the CD5 expression levels in p1positive and p1-negative cells, compared to the bulk. As shown in Figure 3, a higher expression of CD5 was observed in p1-positive cells (representing the VH 1-69 clone) as compared to p1-negative cells (the remaining CLL clones with IgBCR rearrangements differing from VH 1-69), both in CLL1 (Figure 3A) and CLL5 patient (Figure 3B), indicating that CD5 expression levels were related to the expression of VH1-69 rearrangement of IgBCR.

\section{DISCUSSION}

CLL clinical course could be characterized by the presence of different tumor B cell clones that could appear or disappear over time, recognized only by the different variable regions of the expressed IgBCRs. These clonal populations may influence the prognosis of the disease, establishing a balanced condition in which the patient remains stable for many years without therapy requirement, or one of them could escape from the apoptosis and proliferation checkpoints, resulting in tumor progression, the need for therapy, and in some cases fatal outcome.

In this scenario, it is interesting the investigation of molecular mechanisms which allow that particular B cell tumor clones to be more aggressive compared to the other tumor populations coexisting in the same patient.

Several studies were conducted in the field of predicting prognosis factors, including the presence or absence of zetachain-associated protein kinase (ZAP)-70 or CD38 (21), genomic alterations (10), TP53 status (22), and mutational status of the IgBCR (18).

In particular, the mutational status was one of the first prognostic factors evaluated in CLL patients, observing that 
A

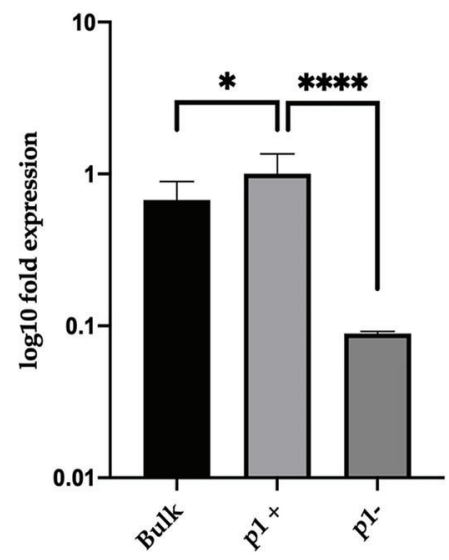

B

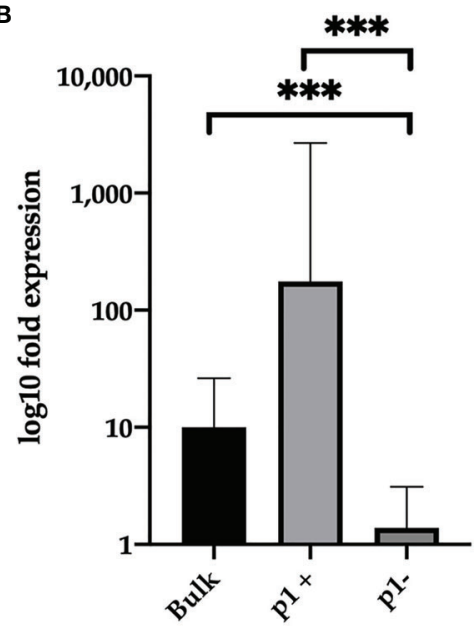

FIGURE 3 | Relative expression of CD5 in p1 positive and p1 negative CLL clones of CLL1 and CLL5 patients. Relative CD5 gene expression in B-CLL subpopulations of CLL1C (A) and CLL5C (B) sample. Bulk represents the total CLL population, p1-positive the sorted VH1-69 CLL cells, and p1-negative the remaining CLL cells. The results, normalized to the GAPDH housekeeping gene and determined by $\triangle \Delta$ Ct method, are represented as log 10 fold expression \pm SD of triplicate assessments. Statistical significance was evaluated using one-way analysis of variance (ANOVA), followed by Bonferroni's test for multiple comparisons. Bars show mean values $\pm 95 \%$ confidence intervals based on three biological replicates. ${ }^{*} P \leq 0.01 ;{ }^{* \star *} P \leq 0.001 ;{ }^{* \star \star *} P \leq 0.0001$.

patients with unmutated immunoglobulins showed a poor prognosis with drug resistance and rebound (18).

The keystone could be peculiar IgBCR rearrangements that result in self-stimulation through binding to intrinsic motifs of the $\operatorname{IgBCR}(14,15)$ or, on the other side, the presence of a persistent stimulation guided and triggered by endogenous and exogenous epitopes (23-25).

Further, the sequence analysis of IgBCRs expressed by CLL cells, compared to normal B cells, revealed the expression of quasi-identical Ig receptors circa in $30 \%$ of diagnosed patients, defined stereotyped IgBCRs (26). These observations fit with the hypothesis of a common exogenous or endogenous antigen stimulating the tumor IgBCRs (14, 15, 23-25).

Reported data describe the capability of the IgBCR to induce the transcription of CD5 by B cells in a murine model (27), which, in turn, transduces pro-survival signaling, such as the IL10 production (28). Furthermore, it was previously demonstrated that circulating CLL cells, that have been activated recently in proliferating centers, express high levels of surface CD5, which is progressively downregulated as the cells enter into an anergy state, suggesting that the CD5 expression levels could be correlated with the aggressive behavior of the CLL cells (29). Thus, CD5 seems to bind to the Ig heavy chain framework sequence of CLL cells, with a preference for the VH1-69 rearrangement (5), which could promote the selective expansion of B cell clones harboring specific VH genes in CLL.

Our study could represent the proof of concept of the potential use of specific peptide ligands of IgBCRs as probes for sorting and analyzing single tumor subpopulations in CLL patients. Thanks to this new methodology, and according to all well-reported data mentioned above, we observed that the CD5 expression level was increased with the expansion of a specific
CLL subpopulation, in our case the unmutated VH1-69 clone, and this could be part of the mechanism of clonal expansion and persistence during the observation, representing additional information for CLL prognosis. More specifically, we demonstrated that single CLL clones could express variable CD5 expression levels, according to their tumorigenic behavior and IgBCR rearrangement.

In perspective, this research line could be extended to all tumor populations of CLL, allowing a wide gene expression analysis, for associating a peculiar IgBCR rearrangement to a specific panel of up or down- regulated genes. We are confident that this approach could get further insights into mechanisms of tumor progression and patient-specific molecular therapy.

\section{DATA AVAILABILITY STATEMENT}

The original contributions presented in the study are included in the article/supplementary materials. Further inquiries can be directed to the corresponding authors.

\section{ETHICS STATEMENT}

Experiments involving human subjects were approved by the Italian Regional "Calabria" Ethics Committee (Protocol N. 75, 23/03/17), in accordance with the ethical and safety rules and guidelines provided by the relevant Italian laws (art. 4-5 of D. lgs 116/92, DD.MM. of 29/09/1995 and 26/04/2000), and in accordance with the ethical guidelines of the European Community Council (directive n. 86/609/ECC). Blood samples from healthy donors or CLL patients were obtained upon written 
and oral informed consent from the participants to the study. The patients/participants provided their written informed consent to participate in this study.

\section{AUTHOR CONTRIBUTIONS}

DM and SM designed and conducted the research, analyzed the data, and wrote the manuscript. EI assisted with the experiment and data analysis. VD designed qRT-PCR primers and protocols and analyzed data. AD'A, FC, and MG provided CLL samples and clinical analysis. MS performed cell sorting. EV, $\mathrm{NN}, \mathrm{AA}, \mathrm{EDS}$, and GF focused on data analysis. IQ and SM

\section{REFERENCES}

1. Boumsell L, Coppin H, Pham D, Raynal B, Lemerle J, Dausset J, et al. An Antigen Shared by a Human T Cell Subset and B Cell Chronic Lymphocytic Leukemic Cells. Distribution on Normal and Malignant Lymphoid Cells. J Exp Med (1980) 152:229-34. doi: 10.1084/jem.152.1.229

2. Agee JF, Garver FA, Faguet GB. An Antigen Common to Chronic Lymphocytic and Hairy Cell Leukemia Cells Not Shared by Normal Lymphocytes or by Other Leukemic Cells. Blood (1986) 68:62-8. doi: 10.1182/blood.V68.1.62.62

3. Padilla O, Calvo J, Vilà JM, Arman M, Gimferrer I, Places L, et al. Genomic Organization of the Human CD5 Gene. Immunogenetics (2000) 993-1001. doi: $10.1007 /$ s002510000235

4. Brown MH, Lacey E. A Ligand for CD5 is CD5. J Immunol (2010) 185:606874. doi: 10.4049/jimmunol.0903823

5. Pospisil R, Silverman GJ, Marti GE, Aruffo A, Bowen MA, Mage RG. CD5 is A Potential Selecting Ligand for B-cell Surface Immunoglobulin: A Possible Role in Maintenance and Selective Expansion of Normal and Malignant B Cells. Leuk Lymphoma (2000) 36:353-65. doi: 10.3109/10428190009148857

6. Jaseb K, Purrahman D, Shahrabi S, Ghanavat M, Rezaeean H, Saki N. Prognostic Significance of Aberrant CD5 Expression in B-Cell Leukemia. Oncol Rev (2019) 13:400. doi: 10.4081/oncol.2019.400

7. Garaud S, Morva A, Lemoine S, Hillion S, Bordron A, Pers JO, et al. CD5 Promotes IL-10 Production in Chronic Lymphocytic Leukemia B Cells Through STAT3 and NFAT2 Activation. J Immunol (2011) 186:4835-44. doi: 10.4049/jimmunol.1003050

8. Raman C. CD5, an Important Regulator of Lymphocyte Selection and Immune Tolerance. Immunol Res (2002) 26:255-63. doi: 10.1385/IR:26:1-3:255

9. Mimmi S, Vecchio E, Iaccino E, Rossi M, Lupia A, Albano F, et al. Evidence of Shared Epitopic Reactivity Among Independent B-cell Clones in Chronic Lymphocytic Leukemia Patients. Leukemia (2016) 30:2419-22. doi: 10.1038/ leu.2016.245

10. Hallek M. Chronic Lymphocytic Leukemia: 2020 Update on Diagnosis, Risk Stratification and Treatment. Am J Hematol (2019) 94:1266-87. doi: 10.1002/ ajh. 25595

11. Kil LP, Yuvaraj S, Langerak AW, Hendriks RW. The Role of B Cell Receptor Stimulation in CLL Pathogenesis. Curr Pharm Des (2012) 18:3335-55. doi: 10.2174/138161212801227041

12. Schleiss C, Ilias W, Tahar O, Güler Y, Miguet L, Mayeur-Rousse C, et al. BCRAssociated Factors Driving Chronic Lymphocytic Leukemia Cells Proliferation Ex Vivo. Sci Rep (2019) 9:701. doi: 10.1038/s41598-018-36853-8

13. Zhang S, Kipps TJ. The Pathogenesis of Chronic Lymphocytic Leukemia. Annu Rev Pathol (2014) 9:103-18. doi: 10.1146/annurev-pathol-020712163955

14. Binder M, Müller F, Frick M, Wehr C, Simon F, Leistler B, et al. Cll B-cell Receptors can Recognize Themselves: Alternative Epitopes and Structural Clues for Autostimulatory Mechanisms in CLL. Blood (2013) 121:239-41. doi: 10.1182/blood-2012-09-454439

15. Dühren-von Minden M, Übelhart R, Schneider D, Wossning T, Bach MP, Buchner M, et al. Chronic Lymphocytic Leukaemia Is Driven by Antigen- supervised the research plan and data analysis, and reviewed the manuscript. All authors contributed to the article and approved the submitted version.

\section{FUNDING}

This work was supported by the following grants: POR FES/ FESR 2014-20-ATS ALCMEONE cup J18C17000610006 to IQ; MIUR-PRIN 2017MHJJ55 002 to IQ; GILEAD Fellowship 2018 to EI. SM was supported by funds from the EU project PONAIM1897004-1; DM was supported by funds from the EU project FSE-FESR PON-RI2014-2020.

Independent Cell-Autonomous Signalling. Nature (2012) 489:309-12. doi: 10.1038 /nature 11309

16. Agathangelidis A, Psomopoulos F, Stamatopoulos K. Stereotyped B Cell Receptor Immunoglobulins in B Cell Lymphomas. Methods Mol Biol (2019) 1956:139-55. doi: 10.1007/978-1-4939-9151-8 7

17. Packham G, Krysov S, Allen A, Savelyeva N, Steele AJ, Forconi F, et al. The Outcome of B-cell Receptor Signaling in Chronic Lymphocytic Leukemia: Proliferation or Anergy. Haematologica (2014) 99:1138-48. doi: 10.3324/ haematol.2013.098384

18. Rotbain EC, Frederiksen H, Hjalgrim H, Rostgaard K, Egholm GJ, Zahedi B, et al. IGHV Mutational Status and Outcome for Patients With Chronic Lymphocytic Leukemia Upon Treatment: A Danish Nationwide Population-Based Study. Haematologica (2020) 105:1621-9. doi: 10.3324/haematol.2019.220194

19. Hamblin TJ, Davis Z, Gardiner A, Oscier DG, Stevenson FK. Unmutated Ig V (H) Genes are Associated With a More Aggressive Form of Chronic Lymphocytic Leukemia. Blood (1999) 94:1848-54. doi: 10.1182/blood. V94.6.1848

20. Mimmi S, Maisano D, Nisticò N, Vecchio E, Chiurazzi F, Ferrara K, et al. Detection of Chronic Lymphocytic Leukemia Subpopulations in Peripheral Blood by Phage Ligands of Tumor Immunoglobulin B Cell Receptors. Leukemia (2021) 35:610-4. doi: 10.1038/s41375-020-0885-y

21. Basabaeen AA, Abdelgader EA, BaHashwan OS, Babekir EA, Abdelateif NM, Bamusa SA, et al. Combined Analysis of ZAP-70 and CD38 Expression in Sudanese Patients With B-Cell Chronic Lymphocytic Leukemia. BMC Res Notes (2019) 12:282. doi: 10.1186/s13104-019-4319-8

22. Morabito F, Gentile M, Monti P, Recchia AG, Menichini P, Skafi M, et al. TP53 Dysfunction in Chronic Lymphocytic Leukemia: Clinical Relevance in the Era of B-cell Receptors and BCL-2 Inhibitors. Expert Opin Investig Drugs (2020) 29:869-80. doi: 10.1080/13543784.2020.1783239

23. Lupia A, Mimmi S, Iaccino E, Maisano D, Moraca F, Talarico C, et al. Molecular Modelling of Epitopes Recognized by Neoplastic B Lymphocytes in Chronic Lymphocytic Leukemia. Eur J Med Chem (2020) 185:111838. doi: 10.1016/j.ejmech.2019.111838

24. Os A, Bürgler S, Ribes AP, Funderud A, Wang D, Thompson KM, et al. Chronic Lymphocytic Leukemia Cells Are Activated and Proliferate in Response to Specific T Helper Cells. Cell Rep (2013) 4:566-77. doi: 10.1016/ j.celrep.2013.07.011

25. Darwiche W, Gubler B, Marolleau JP, Ghamlouch H. Chronic Lymphocytic Leukemia B-Cell Normal Cellular Counterpart: Clues From a Functional Perspective. Front Immunol (2018) 9:683. doi: 10.3389/fimmu.2018.00683

26. Colombo M, Bagnara D, Reverberi D, Matis S, Cardillo M, Massara R, et al. Tracing CLL-Biased Stereotyped Immunoglobulin Gene Rearrangements in Normal B Cell Subsets Using a High-Throughput Immunogenetic Approach. Mol Med (2020) 26:25. doi: 10.1186/s10020-020-00151-9

27. Cong YZ, Rabin E, Wortis HH. Treatment of Murine CD5- B Cells With antiIg, But Not LPS, Induces Surface CD5: Two B-cell Activation Pathways. Int Immunol (1991) 3:467-76. doi: 10.1093/intimm/3.5.467

28. Gary-Gouy H, Harriague J, Bismuth G, Platzer C, Schmitt C, Dalloul AH. Human CD5 Promotes B-Cell Survival Through Stimulation of Autocrine IL10 Production. Blood (2002) 100:4537-43. doi: 10.1182/blood-2002-05-1525 
29. Calissano C, Damle RN, Hayes G, Murphy EJ, Hellerstein MK, Moreno C, et al. In Vivo Intraclonal and Interclonal Kinetic Heterogeneity in B-Cell Chronic Lymphocytic Leukemia. Blood (2009) 114:4832-42. doi: 10.1182/blood-2009-05-219634

Conflict of Interest: The authors declare that the research was conducted in the absence of any commercial or financial relationships that could be construed as a potential conflict of interest.
Copyright (C) 2021 Maisano, Iaccino, D’Ambrosio, Chiurazzi, Dattilo, Scalise, Gentile, Vecchio, Nisticò, Aloisio, De Sensi, Fiume, Quinto and Mimmi. This is an open-access article distributed under the terms of the Creative Commons Attribution License (CC BY). The use, distribution or reproduction in other forums is permitted, provided the original author(s) and the copyright owner(s) are credited and that the original publication in this journal is cited, in accordance with accepted academic practice. No use, distribution or reproduction is permitted which does not comply with these terms. 